\title{
DOUBLE FIELD FLIP COOLING CHANNEL FOR THE NEUTRINO FACTORY
}

\author{
V. Balbekov, V.D. Elvira, P. Lebrun, J-M. Rey, P. Spentzouris, FNAL, Batavia, IL 60510, USA \\ Ed Black, IIT, Chicago, IL 60616, USA
}

\begin{abstract}
A $220 \mathrm{~m}$ long ionization cooling system consisting of three solenoids with two field-flip sections, is proposed as a cooling channel for the neutrino factory. The reduction of transverse emittance is achieved using 87 liquid hydrogen absorbers (30-40 cm long), and 87 ( $2 \mathrm{~m}$ long) $200 \mathrm{MHz}$ linacs. The first flip is performed at relatively small magnetic field, $\mathrm{B}=3 \mathrm{~T}$, to keep the longitudinal motion under control. The field is then increased adiabatically up to $7 \mathrm{~T}$ and a second field flip performed. The cooler was studied and simulated in detail. Preceded by a $16 \mathrm{GeV}$ proton driver, a carbon target, a mini-cooler and a buncher, the system provides about 0.082 muons per incident proton.
\end{abstract}

\section{INTRODUCTION}

The Double Flip (DF) channel [1] is based on long solenoids, and cooling is performed in a constant or adiabatically increasing magnetic field. A uniform solenoid, which contains absorbers and linacs, can only cool in two of the four transverse degrees of freedom: the Larmor radii of all the particles decrease while the positions of their Larmor centers remain unchanged. For an infinitely long channel, if we neglect scattering, the muon helices would therefore shrink to lines, and the resulting beam would be wide but without any angular spread. The magnetic field must be reversed at least once to achieve cooling in four dimensions $\left(p_{x}, p_{y}\right.$, as well as $x$ and $\left.y\right)$. The lengths of the channel sections with positive and negative magnetic field can be chosen to achieve a "canonical" phase ellipse on exit (an ellipse with zero angular momentum and no $x-p_{x}$ correlations).

The major disadvantage of a cooling channel based on long solenoids is the perturbation of the longitudinal motion at the field flip regions. The longitudinal velocity, $V_{z}$, depends on the transverse momentum, $p_{T}$. The RF induced oscillation of $V_{z}$ around an equilibrium value (equal to the phase velocity of the accelerating wave $V_{p h}$ ) is therefore $p_{T}$ dependent too: $E_{e q}=$ $\left(m c / \sqrt{1-V_{p h}^{2} / c^{2}}\right) \sqrt{1+p_{T}^{2} /\left(m^{2} c^{2}\right)}$. After the field flip, $p_{T} \simeq e B r / c$, with $r$ is the particle radius, producing a strong perturbation of the synchrotron oscillations. This effect may be mitigated by using a small magnetic field before and immediately after the first flip region. The field may then grow adiabatically to decrease the $\beta$-function and maximize the cooling. The final beam, however, would have a non-zero angular momentum after an exit from such a system. A second field flip section in the high field region, followed by a short additional cooling section, is therefore necessary to suppress the residual angular momentum. Due to the small beam radius at the end of the third section, the perturbation to the longitudinal motion at the second field flip would be small.

\section{DESCRIPTION OF THE CHANNEL}

The cooling lattice consists of four sections (see Fig 1). The first section contains 20 cooling cells, $2.42 \mathrm{~m}$ long, in an almost constant magnetic field of $B_{z}=3 \mathrm{~T}$ on axis. The Larmor centers of the particle motion are therefore unchanged and the transverse size is constant to first order. Between the first and the second sections there is a $1.02 \mathrm{~m}$ long matching section in which the field changes polarity. In the short region following the field reversal, the centers of the Larmor orbits are displaced and in section 2 the muons, to first order, execute Larmor motion about the solenoid axis. This second section consists of 20 cells, $2.42 \mathrm{~m}$ long, with a field on axis which adiabatically (and linearly) grows from $B_{z}=-3 \mathrm{~T}$ to $-7 \mathrm{~T}$ on axis. Here, the beam is focussed strongly, reducing the $\beta$-function for cooling of both the beam size and the transverse momenta. The third section contains 32 cells, $2.51 \mathrm{~m}$ long (the first two cells are still $2.42 \mathrm{~m}$ long), with a field of $B_{z}=-7 \mathrm{~T}$ on axis. The second field flip region is $1.26 \mathrm{~m}$ long and is followed by the fourth section with 15 cells, $2.51 \mathrm{~m}$ long, with a field of $B_{z}=7 \mathrm{~T}$ on axis.

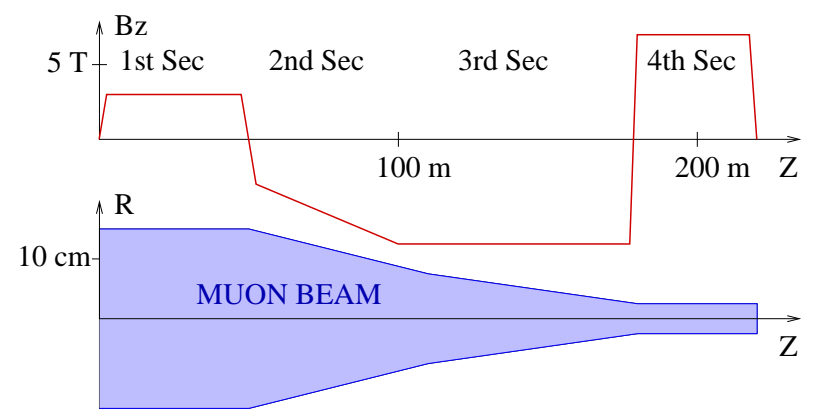

Figure 1: Sketch of $B_{z}$ on axis and the beam envelope associated with the double flip channel.

A cooling cell consists of 6 cavities which provide an effective voltage of $15 \mathrm{MeV} / \mathrm{m}$, and a vessel containing the liquid hydrogen absorber material (LH2).

\subsection{The Magnets}

The long solenoids are $81 \mathrm{~cm}$ in radius, and are designed so that they completely enclose the acceleration cavities. The coil thicknesses vary from $3.5 \mathrm{~cm}$ in the first section, 
to $10.5 \mathrm{~cm}$ in the succeeding sections, as the magnetic field increases. The current densities are 68.2 and $53.1 \mathrm{~A} / \mathrm{mm}^{2}$, respectively. The most sensitive parameter of the cooling channel is the gradient of the magnetic field in the field-flip regions. This gradient must be maximized in order to stabilize the motion. The specifications for the two matching sections are shown in Figs. 2, 3. The $1.02 \mathrm{~m}$ long first flip region consists of two outer coils separated by $20 \mathrm{~cm}$ and two $10.5 \mathrm{~cm}$ long inner coils separated by $26.8 \mathrm{~cm}$ from each other. The $1.26 \mathrm{~m}$ long second flip region consists of the two outer coils separated by $20 \mathrm{~cm}$, and two $24.5 \mathrm{~cm}$ long inner coils separated by $9.6 \mathrm{~cm}$. Both sections achieve a field flip within a distance of less than one meter.

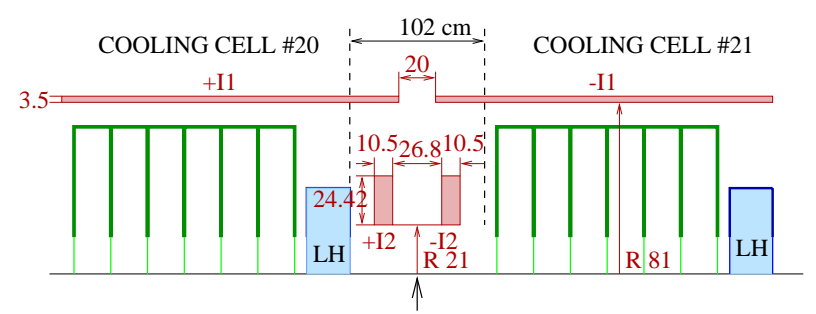

$48.91 \mathrm{~m}$ FROM THE BEGINNING OF THE COOLING CHANNEL

Figure 2: The first matching section.

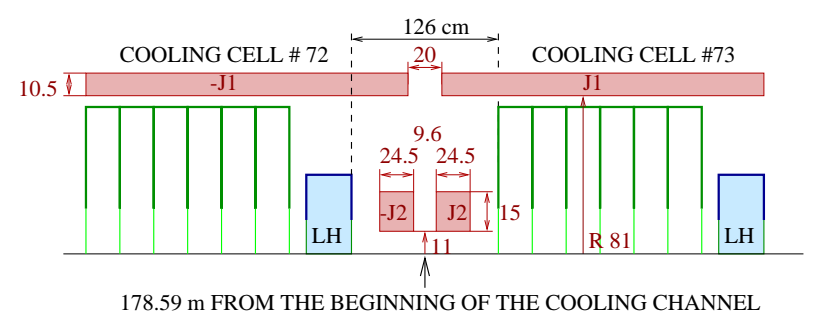

Figure 3: The second matching section.

The design of the solenoidal magnets is based on aluminum stabilized superconductors with indirect cooling. These aluminum stabilized conductors consist of $\mathrm{NbTi}$ super-conducting cables around which an aluminum stabilizer is coextruded.

\subsection{The RF Acceleration}

The RF system consists of one linac per cooling cell, each composed of 6 pill-box copper cavities, $32 \mathrm{~cm}$ long. The radial aperture is $16 \mathrm{~cm}$ in the first two sections of the cooling channel and $7 \mathrm{~cm}$ in the third and fourth. Windows are made of beryllium with thicknesses of $300 \mu \mathrm{m}$ and $25 \mu \mathrm{m}$ respectively. In the first two sections the window thickness follows a step function which goes from $300 \mu \mathrm{m}$ to $600 \mu \mathrm{m}$ at 0.7 the full radius of the window. Thin windows are preferred to reduce the transverse emittance growth due to multiple scattering. Mechanical constraints set a $25 \mu \mathrm{m}$ lower limit to the thickness of a beryllium window.

A peak voltage of $16.48 \mathrm{MV} / \mathrm{m}$, which corresponds to an effective voltage of about $15 \mathrm{MV} / \mathrm{m}$, gives a large enough bucket to capture most of the incoming beam. The beam must be slowly accelerated to enlarge the bucket and compensate for the increase of the energy spread through the channel. A particle with an energy of $200 \mathrm{MeV}$ would lose about $10.4 \mathrm{MeV}$ per cell in the first section and gain $\approx 12.9 \mathrm{MeV}$ per 6-cell linac at a synchronous phase $\phi \approx 25.5^{\circ}$. The nominal energy (defined as equal to the phase velocity of the accelerating wave) is increased to accommodate the bucket size to the growth of the beam in longitudinal phase space. At the beginning and at the end of the channel the nominal energy is $200 \mathrm{MeV}$ and $326 \mathrm{MeV}$, respectively.

\subsection{The Absorbers}

The LH2 absorbers provide the energy loss necessary for the cooling mechanism. The hydrogen is contained by a cryostat vessel. The absorber length is $30 \mathrm{~cm}$ in the first two sections and $39 \mathrm{~cm}$ in the third (after the second cell) and fourth sections. This change compensates for the smaller $d E / d x$ in the material as the energy increases, and approximately maintains the same synchronous phase throughout the channel. The aluminum windows have the same radius as the beryllium windows in the linac, but they must be thick enough to withstand the $1.2 \mathrm{~atm}$ pressure from the LH2. The windows are therefore $360 \mu \mathrm{m}$ and $220 \mu \mathrm{m}$ thick for a radius of $16 \mathrm{~cm}$ and $7 \mathrm{~cm}$ respectively.

\section{THE INPUT BEAM}

The double-flip channel is optimized to maximize the transmission and the cooling performance for the input beam produced at the end of the neutrino source precooling system. The pre-cooling apparatus, and consequently the input beam for the double-flip channel, follows the design in Ref. [2]). The basic components are a $16 \mathrm{GeV}$ proton driver, a solid carbon target, a mini-cooler and a buncher.

The performance of the cooling channel was tested using a "realistic" beam produced by a simulation of the pre-cooling section [1]. The initial parameters are: $\sigma_{x}=54.6 \mathrm{~mm}, \sigma_{p_{x}}=24.4 \mathrm{MeV} / \mathrm{c}, \mu / p=0.191$ (or 0.135 muons per proton inside the initial bucket).

\section{PERFORMANCE}

The performance of the channel was studied with a GEANT4 [3] simulation using the "realistic" beam (a) described in Sec. 3 (4500 particles) [4]. (GEANT4 performs tracking of muons through electromagnetic fields and matter.) The evolution of the channel parameters is illustrated in Fig. 4. The final transverse and longitudinal emittances $\left(\varepsilon_{x}\right.$ and $\varepsilon_{z}$ ) are $1.92 \mathrm{~mm}$ and $75.1 \mathrm{~mm}$, respectively. The longitudinal emittance increases by a factor of 1.6 between the end of the first section and the end of the channel. Both $\sigma_{x}$ and $\sigma_{p_{x}}$ are to to final values of $14.1 \mathrm{~mm}$ and 14.5 MeV/c, respectively. The transmission predicted by the GEANT4 simulation, referred to the total number of 
particles in the initial beam (with a $\mathrm{E}<300 \mathrm{MeV}$ cut), is $43 \%$. In other words, the number of muons per proton, $\mu / \mathrm{p}$, is 0.082 . If we upgraded the proton driver to $24 \mathrm{GeV}$, and the carbon target to a liquid mercury target (as the neutrino source design in Ref. [5]), the yield would increase to $\simeq 0.23$ muons per proton.

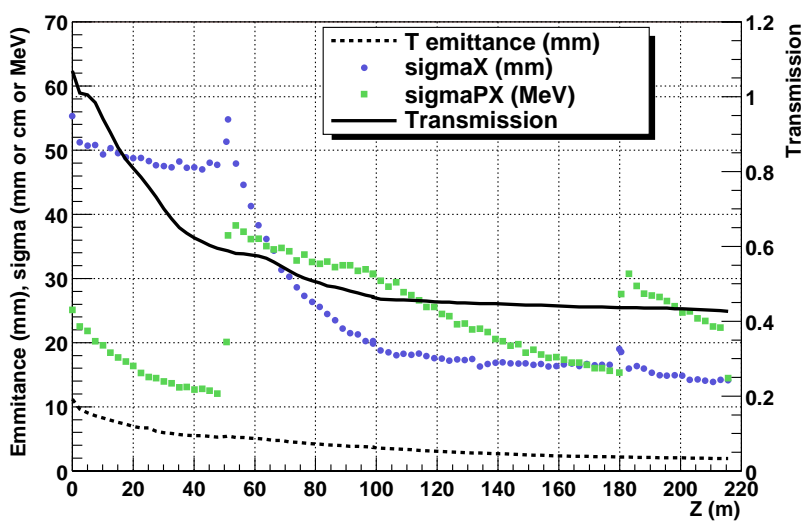

Figure 4: Performance of the Double Flip cooling channel with a "realistic" initial beam. The parameters at the end of the channel are calculated on exit (outside the solenoid).

Figure 5 shows the $E-\langle E\rangle$ versus $c t-\langle c t\rangle$ distribution at the beginning (bottom left) and at the end (bottom right) of the channel. It also illustrates on transverse cooling. The top left plot is the initial $p_{x}$ versus $x$ distribution and the top right plot is the same distribution at the end of the channel. The bunch fills the 201.25 MHz RF bucket from the start. About half of the lost particles are muons that are not captured into the bucket. The remaining particle loss is due to the excitation of longitudinal motion in the first field-flip region. Low-momentum muons are lost at the maximum of the synchrotron oscillation, which is a few meters after the matching section.

For comparison, a second GEANT4 study (b) [4] was done using the same beam through a modified version of the channel which follows more closely the design proposed and simulated (with a simpler model) in Ref. [1]. In (b), only the windows are different: a radius and thickness of $20 \mathrm{~cm}$ and $125 \mu \mathrm{m}$ for the aluminum windows in the LH2 vessels, and a radius and thickness of $20 \mathrm{~cm}$ $(10 \mathrm{~cm})$ and $400 \mu \mathrm{m}(200 \mu \mathrm{m})$ for the beryllium windows in the RF cavities. The parameters at the end of the channel for (b) (and Ref. [1]) are in reasonable agreement: $\sigma_{x}=13.8 \mathrm{~mm}(14.5 \mathrm{~mm}), \sigma_{p_{x}}=15 \mathrm{~mm}(15.4 \mathrm{~mm})$, $\varepsilon_{x}=1.90 \mathrm{~mm}(2.12 \mathrm{~mm}), \varepsilon_{z}=71 \mathrm{~mm}(74.6 \mathrm{~mm})$. Transmission is $47 \%$ and $52 \%$ for (b) and Ref. [1], respectively. Table 1 summarizes the beam parameters at the end of the cooling channel for (a), (b), and Ref. [1].

\section{SUMMARY}

We have developed a conceptual and engineering design of a double flip cooling channel, and studied its performance for a "realistic" beam. A final $\mu / \mathrm{p}$ yield of 0.082
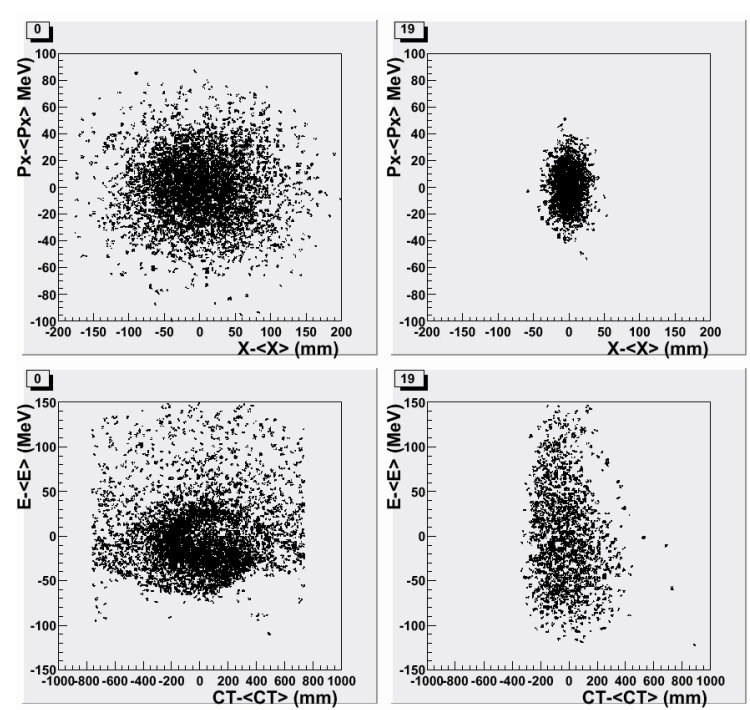

Figure 5: Top (bottom): $p_{x}$ versus $x(E-\langle E\rangle$ versus $c t-$ $\langle c t\rangle)$ at the beginning, left, and the end, right, of the cooling channel.

Table 1: Parameters of the beam at the end of the cooling channel.

\begin{tabular}{|l|l|l|l|l|l|}
\hline Case & $\begin{array}{l}\sigma_{x} \\
(\mathbf{m m})\end{array}$ & $\begin{array}{l}\sigma_{p_{x}} \\
(\mathbf{M e V} / \mathbf{c})\end{array}$ & $\begin{array}{l}\varepsilon_{x} \\
(\mathbf{m m})\end{array}$ & $\begin{array}{l}\varepsilon_{z} \\
(\mathbf{m m})\end{array}$ & $\begin{array}{l}\text { Trans } \\
\%\end{array}$ \\
\hline $\mathbf{( a )}$ & 14.1 & 14.5 & 1.92 & 75.1 & 43 \\
\hline $\mathbf{( b )}$ & 13.8 & 15.0 & 1.90 & 71.0 & 47 \\
\hline Ref. [1] & 14.5 & 15.4 & 2.12 & 74.6 & 52 \\
\hline
\end{tabular}

was obtained for a neutrino factory design which includes a $16 \mathrm{GeV}$ proton driver and a carbon target. If we upgraded the system to a $24 \mathrm{GeV}$ proton driver and a liquid mercury target the number of muons per proton would almost triple to 0.23 .

\section{REFERENCES}

[1] "Double Field Flip Cooling Channel for Neutrino Factory (front-end simulation)", MuCool note \#118, April 2000.

[2] The Muon Collider/Neutrino Source Collaboration, "A Feasibility Study of a Neutrino Source based on a Muon Storage Ring" (based on a Fermilab site). Submitted to Phys. Rev. ST - Accelerators and Beams. FERMILAB-PUB00-108-E (2000).

[3] HTTP://WWWINFO.CERN.CH/ASD/GEANT4/GEANT4.HTML

[4] "The Double Flip Cooling Channel", V. Balbekov, V. D. Elvira, et al. MuCool note \#203, 8/6/00.

[5] The document on a feasibility study of a neutrino source in Brookhaven National Laboratory is currently in preparation.

$\mathrm{MuCool}$ notes are available at

http://www-mucool.fnal.gov/notes/notes.html 